\title{
Corrigendum: Capacity limits of spatially multiplexed free-space communication
}

Ningbo Zhao, Xiaoying Li, Guifang Li and Joseph M. Kahn

Nature Photonics 9, 822-826 (2015); published online 16 November 2015; corrected after print 27 November 2015.

In the version of this Letter originally published, in Fig. 3a,b, the $x$ axis labels should have been 'SNR (dB)'. This has now been corrected in the online versions of the Letter. 